\title{
An Uncommon Presentation of Extrahepatic Cholestasis due to Single Biliary Stricture From Kaposi Sarcoma
}

\author{
Estefanía Flores Córdova ${ }^{1}$, Madhu Mathew ${ }^{2}$, Hemant Mutneja ${ }^{2}$, Elena Gonzalez Caldito ${ }^{2}$, Melchor \\ Demetria $^{3}$
}

\begin{abstract}
1. Internal Medicine, Cook County Health and Hospital System, Chicago, USA 2. Internal Medicine, John H. Stroger, Jr. Hospital of Cook County, Chicago, USA 3. Gastroenterology and Hepatology, John H. Stroger, Jr. Hospital of Cook
\end{abstract} County, Chicago, USA

Corresponding author: Estefanía Flores Córdova, estefaniaflorescordova@gmail.com

\begin{abstract}
Kaposi sarcoma is one of the acquired immunodeficiency syndrome (AIDS) defining diseases. AIDSassociated Kaposi sarcoma affects primarily the skin and the lungs. Although gastrointestinal involvement is relatively common, biliary tract involvement has rarely been reported. It has been associated mostly with extension from liver disease. We describe an uncommon presentation of disseminated Kaposi sarcoma causing extrahepatic cholestasis due to extrahepatic biliary tract involvement that resolved after sphincterotomy with biliary stenting.
\end{abstract}

We present a case of a 35-year-old African American male diagnosed with human immunodeficiency virus (HIV) infection in 2005. He presented with AIDS after discontinuation of antiretroviral therapy for one year, subsequently being diagnosed with systemic Kaposi sarcoma. He presented with signs and symptoms of obstructive biliary disease, including jaundice, abdominal pain, fatigue, and fever. We encountered a rare presentation of malignant single extrahepatic biliary stenosis secondary to biliary Kaposi sarcoma. The biochemical pattern markedly improved after endoscopic retrograde cholangiopancreatography with sphincterotomy and stenting. However, and despite the resumption of combined antiretroviral therapy, deep immunosuppression caused worsening clinical condition and death five months after initial presentation.

Certainly, among the multiple etiologies of biliary obstruction in AIDS, Kaposi sarcoma is one to consider.

Categories: Gastroenterology, HIV/AIDS, Infectious Disease

Keywords: extrahepatic cholestasis, acquired immunodeficiency syndrome, biliary tract, case report, kaposi sarcoma

\section{Introduction}

Received 05/15/2020

Review began 06/02/2020 Review ended 06/24/2020 Published 06/29/2020

\section{() Copyright 2020}

Flores Córdova et al. This is an open access article distributed under the terms of the Creative Commons Attribution License CC-BY 4.0., which permits unrestricted use, distribution, and reproduction in any medium, provided the original author and source are credited.
Kaposi sarcoma (KS) is a low-grade vascular tumor associated with human herpesvirus 8 (HHV-8) infection [1,2]. It was first described in 1872 by Moritz Kaposi, a Hungarian dermatologist, initially affecting mostly Mediterranean or Jewish older men. But it was not until 1981 when a demographic change was discovered by Alvin Friedman Kein proving its association with HIV infection [3-5]. There are four variants of its presentation: classic, endemic, posttransplant, and, lastly, the acquired immunodeficiency syndrome (AIDS) associated or epidemic [1,3,5]. The AIDS-associated presentation was for many years the most common AIDS-associated tumor in the United States [6]. Even though KS can develop at any HIV infection stage, generally it occurs in the setting of advanced immune suppression [7]. The incidence has decreased to less than $1 \%$ of patients with AIDS after the introduction of combined antiretroviral therapy (cART) [6]. AIDS-associated KS is rapidly progressive and known to initially affect the skin; however, it can extend to mucous membranes and internal organs $[1,2,8]$. Gastrointestinal involvement is the most common extracutaneous site $[2,7]$. It is reported that KS can affect any part of the gastrointestinal tract [8]. However, biliary tract involvement has rarely been reported. We present a case with an uncommon presentation of disseminated KS invading the biliary tract and causing extrahepatic cholestasis.

\section{Case Presentation}

We present the case of a 35-year-old African American male with HIV diagnosed in 2005, initially treated with emtricitabine and tenofovir, although due to lack of insurance he postponed cART for one year. He was admitted to our emergency department with persistent fever, generalized abdominal pain, and jaundice for two weeks and a weight loss of 15 pounds in four weeks. Physical examination on admission showed scleral icterus, small hyperpigmented lesions throughout his trunk, a violaceous nodular enlargement of the hard palate, left axillary lymphadenopathy, a diffusely tender abdomen, and hepatomegaly. Laboratory studies were significant for platelets of $75 \mathrm{k} / \mathrm{uL}(161-369 \mathrm{k} / \mathrm{uL})$, new-onset conjugated hyperbilirubinemia with a total bilirubin of $23 \mathrm{mg} / \mathrm{dL}(0.2-1.2 \mathrm{mg} / \mathrm{dL})$, direct bilirubin of $16 \mathrm{mg} / \mathrm{dL}(0.0-0.2 \mathrm{mg} / \mathrm{dL})$, alkaline phosphatase of $288 \mathrm{IU} / \mathrm{L}$ (20-120 IU/L), gamma-glutamyl transferase of $193 \mathrm{IU} / \mathrm{L}$ (3-60 IU/L), aspartate aminotransferase of $66 \mathrm{IU} / \mathrm{L}$ (0-40 IU/L), alanine aminotransferase of $40 \mathrm{IU} / \mathrm{L}$ (5-35 IU/L), and international 


\section{Cureus}

normalized ratio of 1.3. Computed tomography (CT) of the abdomen revealed hepatomegaly and an illdefined heterogeneous mass in the peri-portal area with obstruction of the biliary tract with intrahepatic biliary dilation (Figure 1). Endoscopic retrograde cholangiopancreatography demonstrated a non-stenotic erythematous major papilla and a 3-cm stricture in the common hepatic duct (Figure 2). Sphincterotomy and balloon dilation of the stricture were performed, biopsies were taken, and two 7-Fr 12-cm plastic stents were deployed in the right and left hepatic ducts bypassing the stricture. Esophagogastroduodenoscopy with endoscopic ultrasound revealed an erythematous 15-mm nodule in the cardia (Figure 3), with cold biopsies taken and subsequent fine needle biopsy of the peri-portal area performed as well. Inguinal lymph node needle core node biopsy was also performed. Pathology from the porta hepatis mass showed blood clot; however, immunohistochemical staining of the common hepatic duct (Figure 4), stomach nodule (Figure 5), and inguinal lymph node biopsies were positive for HHV-8 KS. cART was commenced, and doxorubicin therapy was started after liver function tests showed improvement. The patient was discharged in view of symptomatic improvement. Liver function tests normalized eight weeks after initial presentation. Nevertheless, his health deteriorated, presenting with hemoptysis, bronchoscopy showed diffuse pulmonary KS, and, subsequently, he had recurrent multiple admission for healthcare-associated pneumonia, cytomegalovirus pneumonitis, pulmonary embolism, and acute tubular necrosis. The patient expired five months after his initial presentation.

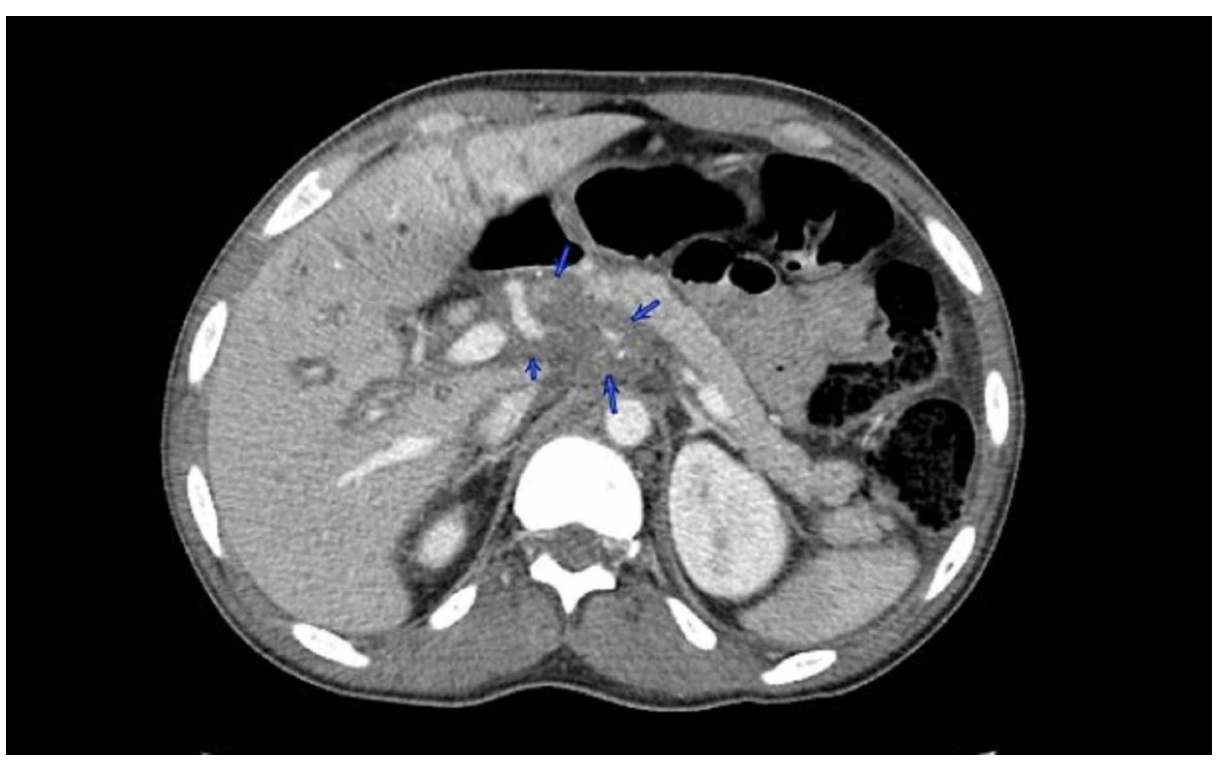

\section{FIGURE 1: CT of the abdomen}

CT of the abdomen showing an infiltrative process in the portal hepatis to the celiac area (blue arrows) with obstruction of the biliary tract with intrahepatic biliary dilation.

CT, computed tomography 


\section{Cureus}

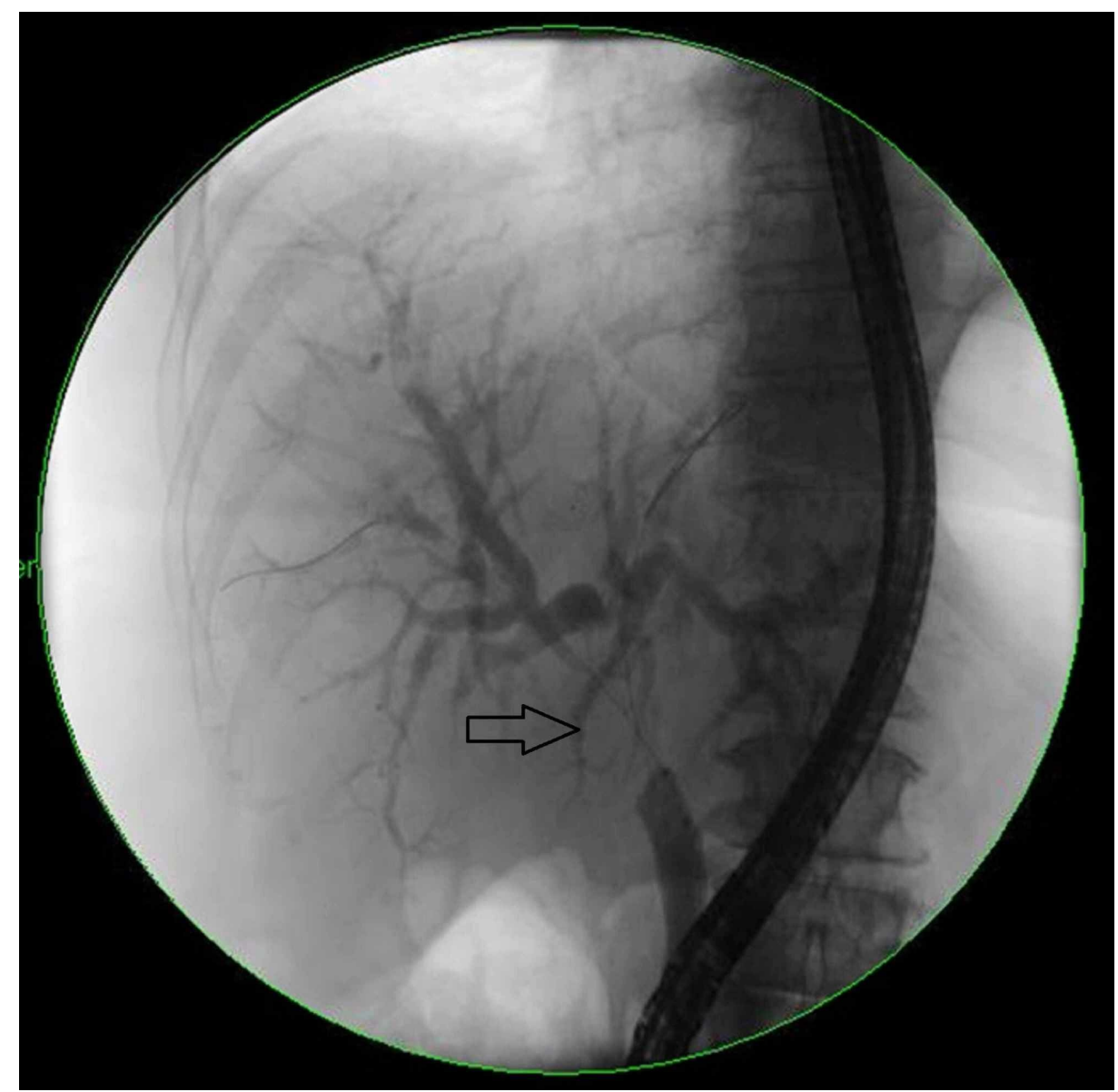

FIGURE 2: ERCP

Common hepatic duct with a 3-cm stricture (black arrow).

ERCP, endoscopic retrograde cholangiopancreatography

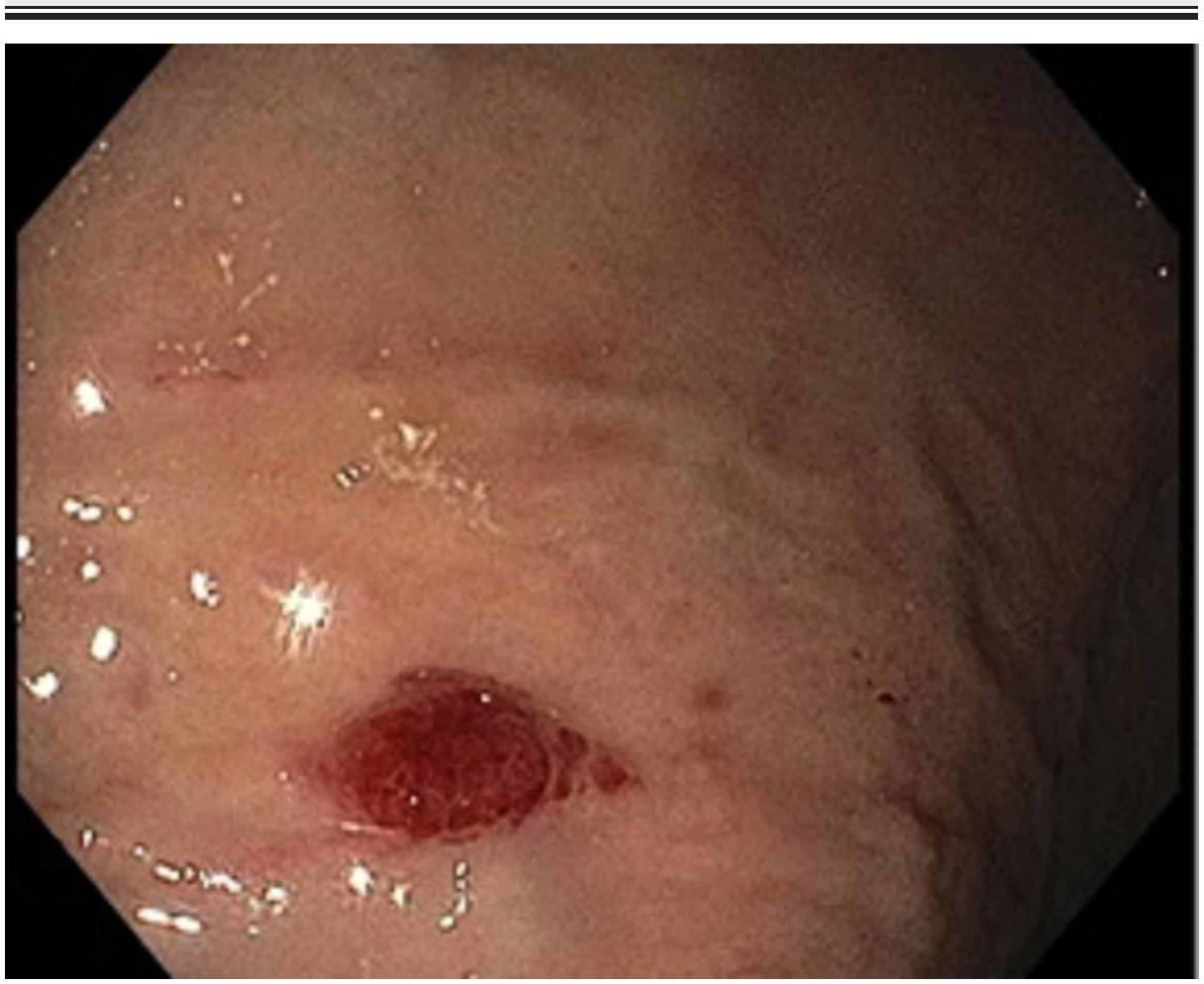




\section{Cureus}

\section{FIGURE 3: Stomach nodule}

Erythematous 15-mm nodule in the stomach cardia evidenced during EGD.

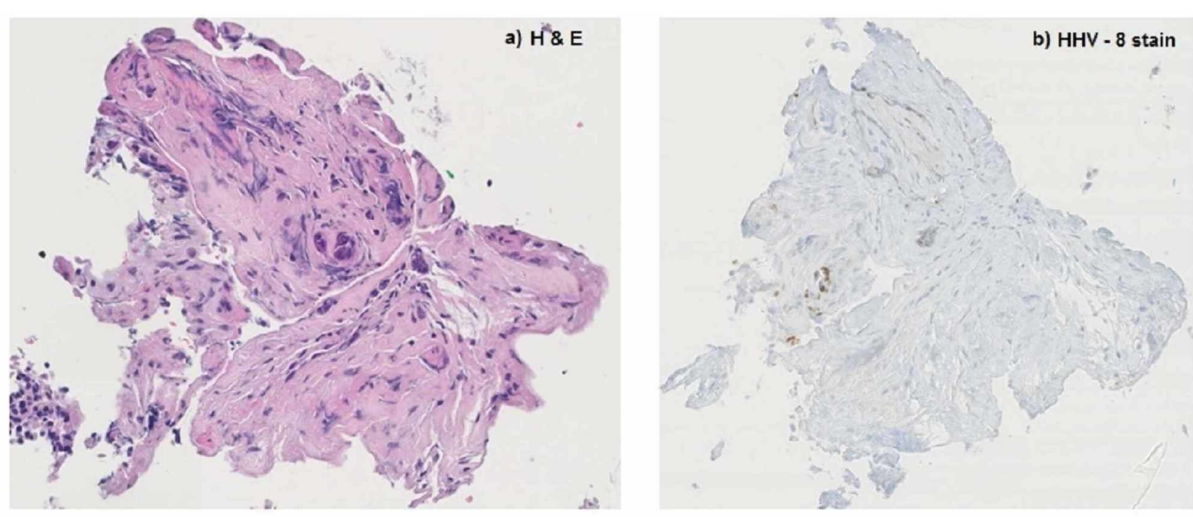

\section{FIGURE 4: Common hepatic duct biopsy}

H\&E stain (a): benign biliary epithelium and scant fibrous fragments, fibrin, and blood. Immunostain for HHV-8 (b): few endothelial cell nuclei are immunoreactive.

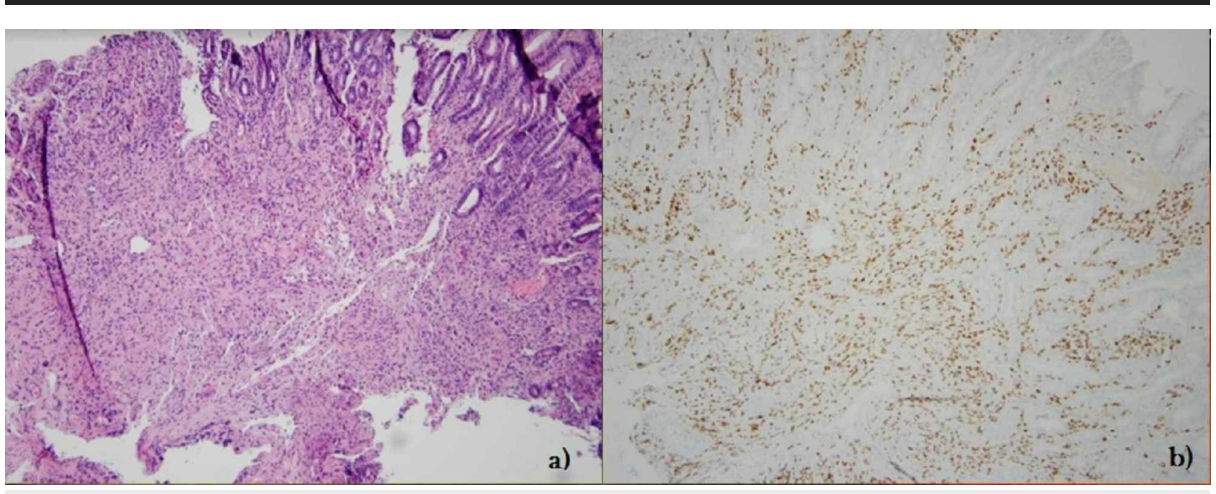

\section{FIGURE 5: Stomach nodule biopsy}

H\&E stain (a): Kaposi sarcoma involving gastric mucosa. (b): Immunostain for HHV-8 is diffusely positive in the endothelial cell proliferation.

H\&E, hematoxylin and eosin; HHV-8, human herpesvirus 8

\section{Discussion}

Gastrointestinal KS is generally asymptomatic in two-thirds of the cases, and bleeding might be the initial presentation $[7,9]$. Some patients can present with nonspecific symptoms such as abdominal pain, weight loss, malabsorption, and diarrhea, like the initial clinical presentation of our patient $[8,9]$.

KS involving the biliary tract is infrequent and rarely reported, and an extensive literature review revealed only a few cases. KS has been associated mostly with extension from liver disease presenting with cholangitis and jaundice and may also be caused by extensive disease of the porta hepatis with compression of the extrahepatic biliary tree [10]. A Spanish journal reported a case of biliary tract and gallbladder KS in an AIDS patient without cutaneous involvement that initially presented with pancreatitis; in this case, the involvement of the biliary tract appeared similar to primary sclerosing cholangitis given the beaded pattern [11]. Another study reported a case of a patient with marked changes of sclerosing cholangitis of the intrahepatic bile ducts with postmortem diagnosis of diffuse hepatic KS that caused infiltration of the bile duct [12]. However, our patient had one single 3-cm stricture in the common hepatic duct, which was 
cannulated with complete resolution of the obstructive pattern. Our patient did not have signs of hepatic involvement nor infiltration extending from the liver. This type of presentation has not been described in the medical literature previously.

Our patient presentation might also be explained by AIDS cholangiopathy, a form of biliary tract inflammation with stricture formation in severely immunosuppressed HIV patients $[13,14]$. Although the identification of HHV-8 through immunostaining in the tissue biopsy obtained from the biliary tract makes the diagnosis of biliary KS more likely, cutaneous or visceral biopsy is required for the diagnosis of KS. Histopathological features include vascular or spindle cell formations and inflammatory infiltration. Immunohistochemical staining is characteristic of HHV-8 expression, as demonstrated in the common hepatic duct biopsy of our patient [2]. In paraffin-embedded sections, HHV-8 immunostaining has a sensitive (99\%) and specific (100\%) [8,15].

In patients with advanced symptomatic cutaneous or extracutaneous disease, the role of chemotherapy in addition to standard antiretroviral therapy has been demonstrated to reduce disease progression $[2,16]$. The current first-line treatment for advanced KS according to JNCCN (Journal of the National Comprehensive Cancer Network) guidelines is liposomal doxorubicin. An alternative option for first-line systemic therapy is paclitaxel [17]. Patients treated with pegylated liposomal doxorubicin or paclitaxel have shown clinical benefit and tumor response, but their side effects are not negligible [16,18]. Newer targeted treatments are being explored; however, the declining incidence of AIDS-related KS makes it difficult to create large-scale trials [19]. HHV-8 infection cannot be eradicated, and complete remission in the setting of advanced disease at presentation is rare; however, reduction or reversion of symptoms and mitigation of end-organ damage can be achieved [17].

As treatment of HIV has improved, a reduction in the incidence of HIV-associated KS has been evidenced; however, in patients without treatment or interrupted therapy, especially those with AIDS, high level of suspicion should be maintained to timely diagnose HHV-8 infection, treat accordingly, and avoid future complications $[17,20]$. Even though our patient's symptoms and chemistry improved initially, his marked immunosuppression triggering multiple opportunistic infections caused his death.

\section{Conclusions}

KS is an opportunistic malignancy seen commonly in patients with AIDS and can frequently involve the gastrointestinal tract. Any part of the gastrointestinal tract can be affected by KS. Isolated biliary stricture is rarely reported; however, it must be considered among the multiple etiologies of biliary obstruction in AIDS.

\section{Additional Information \\ Disclosures}

Human subjects: Consent was obtained by all participants in this study. Conflicts of interest: In compliance with the ICMJE uniform disclosure form, all authors declare the following: Payment/services info: All authors have declared that no financial support was received from any organization for the submitted work. Financial relationships: All authors have declared that they have no financial relationships at present or within the previous three years with any organizations that might have an interest in the submitted work. Other relationships: All authors have declared that there are no other relationships or activities that could appear to have influenced the submitted work.

\section{References}

1. Radu O, Pantanowitz L: Kaposi sarcoma. Arch Pathol Lab Med. 2013, 137:289-294. 10.5858/arpa.2012-0101RS

2. Leer-Greenberg BV, Kole A, Chawla S: Hepatic Kaposi sarcoma: a case report and review of the literature . World J Hepatol. 2017, 9:171-179. 10.4254/wjh.v9.i4.171

3. Sternbach G, Varon J: Moritz Kaposi: idiopathic pigmented sarcoma of the skin . J Emerg Med. 1995, 13:671674. 10.1016/0736-4679(95)00077-N

4. Cohen JM, Burgin S. : Moritz Kaposi: a notable name in dermatology . JAMA Dermatol. 2015, 151:867. 10.1001/jamadermatol.2015.1075

5. Millet A, Singh S, Gittens-Backus G, Dang KA, Shokrani B: An unusual case of invasive Kaposi's sarcoma with primary effusion lymphoma in an HIV positive patient. Case Rep Oncol Med. 2015, 2015:789616. $10.1155 / 2015 / 789616$

6. Tacconi D, Vergori A, Lapini L, Magnolfi A, Carnevali A, Caremani M: Hepatic Kaposi's sarcoma in a patient affected by AIDS: correlation between histology and imaging. J Ultrasound. 2012, 15:215-219. 10.1016/j.jus.2012.10.004

7. Lee AJ, Brenner L, Mourad B, Monteiro C, Vega K, Munoz J: Gastrointestinal Kaposi's sarcoma: case report and review of the literature. World J Gastrointest Pharmacol Ther. 2015, 6:89-95.

8. Zapata Laguado MI, Aponte Monsalve JE, Santos JH, Preciado J, Mosquera Zamudio A, Garza Acosta C: Primary gastrointestinal Kaposi's sarcoma in a patient with human immunodeficiency virus . Case Rep Oncol. 2018, 11:638-647. 10.1159/000492715

9. Arora M, Goldberg EM: Kaposi sarcoma involving the gastrointestinal tract. Gastroenterol Hepatol (N Y). 2010, 6:459-462. 


\section{Cureus}

10. Goldin RD, Hunt J: Biliary tract pathology in patients with AIDS . J Clin Pathol. 1993, 46:691-693.

11. Segarra P, Abril V, Gil M, Ortega E, Ballester JE, Traves V: [Kaposi's sarcoma of the bile ducts without cutaneous involvement in a patient with AIDS]. Rev Esp Enferm Dig. 1996, 88:637-639.

12. Cello JP: Acquired immunodeficiency syndrome cholangiopathy: spectrum of disease . Am J Med. 1989, 86:539-546. 10.1016/0002-9343(89)90381-1

13. Kohli DR, Lippman HR, Shah TU: A patient with AIDS and an unusual cause of jaundice . Gastroenterology. 2017, 152:11-12. 10.1053/j.gastro.2016.11.017

14. Gao Y, Chin K, Mishriki YY: AIDS cholangiopathy in an asymptomatic, previously undiagnosed late-stage HIV-positive patient from Kenya. Int J Hepatol. 2011, 2011:465895. 10.4061/2011/465895

15. Robin, YM, Guillou L, Michels JJ, Coindre JM: Human herpesvirus 8 immunostaining: a sensitive and specific method for diagnosing kaposi sarcoma in paraffin-embedded sections. Am J Clin Pathol. 2004, 121:330-334. 10.1309/96U16LRRAN5HWWVE

16. Cooley T, Henry D, Tonda M, Sun S, O’Connell M, Rackoff W: A randomized, double-blind study of pegylated liposomal doxorubicin for the treatment of AIDS-related Kaposi's sarcoma. Oncologist. 2007, 12:114-123. 10.1634/theoncologist.12-1-114

17. Reid E, Suneja G, Ambinder RF, et al.: AIDS-Related Kaposi Sarcoma, Version 2.2019, NCCN Clinical Practice Guidelines in Oncology. J Natl Compr Canc Netw. 2019, 17:171-189. 10.6004/jnccn.2019.0008

18. Cianfrocca M, Lee S, Von Roenn J, et al.: Randomized trial of paclitaxel versus pegylated liposomal doxorubicin for advanced human immunodeficiency virus-associated Kaposi sarcoma: evidence of symptom palliation from chemotherapy. Cancer. 2010, 116:3969-3977. 10.1002/cncr.25362

19. Cesarman E, Damania B, Krown SE, Martin J, Bower M, Whitby D: Kaposi sarcoma. Nat Rev Dis Primers. 2019, 5:9. 10.1038/s41572-019-0060-9

20. Goncalves P, Uldrick T, Yarchoan R: HIV-associated Kaposi sarcoma and related diseases. AIDS. 2017, 31:1903-1916. 10.1097/QAD.0000000000001567 